\title{
EVTM After COVID
}

\author{
Rishi Kundi, Jonathan Morrison and Thomas Scalea
}

R. Adams Cowley Shock Trauma Center, Baltimore, MD, USA

The start of the 2021 EVTM Symposium last month came more than two years after the Pan-American meeting in Denver. For many of us, it was the first time we had traveled internationally since that meeting. For all of us, it was a long-awaited reunion of colleagues.

During that interval, medicine seemed to evolve to be COVID and nothing but COVID. Even the contents of medical journals hinted that the novel coronavirus was the only game in town, regardless of the specialty or topics typically covered. Yet other medical problems continued. In Baltimore, every day of the past two years we cared for victims of motor vehicle collisions, shootings, stabbings, and the usual improbable accidents. Trauma volumes fell for the first six weeks of the pandemic and then rebounded with enthusiasm - particularly trauma from interpersonal violence.

Sadly, Baltimore will likely set yet another per capita record for homicides in 2021. Given the sophistication of the Maryland Trauma System, one can only postulate how much worse it could be without this system. The strain of the pandemic has made rapid resuscitation and treatment even more difficult, but the trauma community everywhere showed up, working around the clock and around the limitations of the pandemic, and did the best they could.

The pandemic and its response provide a clear lesson for the EVTM constituency. When presented with a universal threat from unknown pathology, medicine scrambled to investigate, respond, and devise the best

\section{Corresponding author:}

Rishi Kundi, Assistant Professor of Surgery, University of Maryland School of Medicine, Programs in Trauma, Vascular/ Endovascular Trauma, and Critical Care, R. Adams Cowley Shock Trauma Center, University of Maryland Medical Center, Baltimore, MD, USA.

Email:RKundi@som.umaryland.edu

(C) 2021 CC BY 4.0 - in cooperation with Depts. of Cardiothoracic/ Vascular Surgery, General Surgery and Anesthesia, Örebro University Hospital and Örebro University, Sweden possible treatment. The omnipresent turf wars went away. There were no society statements proclaiming that COVID pneumonia was properly the province of the intensivist. Pulmonologists did not angrily declaim that hematologists had no business managing COVID coagulopathy.

Instead, there was a mad rush to collaborate. Information was prized and those who found it celebrated, regardless of their field. In academic institutions, community medical centers, and ad hoc field hospitals, nephrologists were given crash courses in ventilator management and family practice doctors refreshed their central line techniques. For the last two years, medicine has not been about domain and territory; it has been about having someone at the bedside when someone is needed at the bedside. It has been about having a set of skills when those skills were needed.

Our motto - "no ego, just good science and collaboration" - could easily have been that of our COVID response. As the world starts to resume its routines, the discussions and debates about trauma and resuscitation care have picked up again. Commentaries, responsa, and letters to the editors debate who is best equipped to care for these patients. Compared to those before the pandemic, these claims are no longer laced with righteous indignation. Instead of stubborn flag-planting, the pieces end with an assertion that cooperation is needed, that the way forward is together. Perhaps the pandemic has reminded all of medicine that we must stand, and work, together.

At Shock Trauma, we have been pleased to be at the forefront of the use of endovascular care for trauma. We pride ourselves in harnessing collaborative expertise from diverse practitioners from many disciplines when caring for the sickest and most complex patients in the United States. We were honored to present a session in Sweden and hope it was useful to the audience. In this spirit, we are pleased to announce that the 2022 EVTM Symposium will be held in Baltimore, Maryland. The R. Adams Cowley Shock Trauma Center looks forward to hosting all of you and to further discussions about the future of endovascular trauma care and resuscitation. 